100 years, until Poincaré demonstrated the divergence of the series used by astronomers.

The work of Poisson and Haretu belongs to this quest. Their goal was to compute the higher-order terms in the semi-major axis variations. Poisson demonstrated that, as a second-order approximation with respect to the masses, the semi-major axes do not present purely secular terms but instead have mixed terms. Then Haretu showed in his thesis the possibility of secular terms to third order with respect to the masses.

I find it hard to support the authors' enthusiastic commentary on Haretu's work: "Haretu's theorem can be considered as a foundation stone for the great edifice of KAM theory". Unlike Poisson's results, which inspired Poincaré's famous recurrence theorem, Haretu's results do not appear to have had any profound repercussions. And as a response to a decades-old question, the implications of his admittedly important findings are limited: the secular terms found by Haretu are simply the Taylor expansion of some small periodic terms due to the quasiperiodic variations of the eccentricities as they appear in Laplace's solutions. The possibility of instabilities in the Solar System does not result from Haretu's work but from Poincaré's demonstration of the divergence of the series of celestial mechanics.

Towards the end of the book, the authors suggest that Xia's recent results on Arnold diffusion in the three-body problem could help to explain the Kirkwood gaps in the asteroid belt. I cannot agree.

First of all, the authors seem to forget that recent extensive work using numerical techniques and perturbation theory now provides a virtually complete view of the dynamics of the asteroid belt. It is true that these results are not "rigorous mathematical proofs", but then again the Solar System is not a mathematical object. Moreover, most of these results can be related locally to small models for which the dynamical behaviour is relatively well understood. It is therefore unfair to disregard these works.

On the other hand, Xia's results apply only to a caricature of the Solar System, require unrealistically small perturbations and result in extremely bad time-scales for the diffusion. Although these results (if vindicated mathematically — the proofs are disputed) may be a remarkable mathematical achievement, they seem to provide no better insight into the physical understanding of diffusion in the asteroid belt than does Arnold's original result of 1964.

Diacu and Holmes confront a difficult challenge in aiming to provide an accessible account of celestial mechanics as the origin of many of the ideas in chaos theory. In doing so, they seem driven to taking certain liberties with the strictly historical account. In particular, many of the story's characters are given thoughts they probably never had, and are generally portrayed as behaving like modern US scientists.

Despite these shortcomings and some obvious subjectivity in the narration, this book is welcome for its detailed account of the resolution of Painlevé's conjecture. It will provide the reader with a lively introduction to the fascinating story of celestial mechanics. $\square$ Jacques Laskar is at Astronomie et Systèmes Dynamiques, Bureau des Longitudes, 3 rue Mazarine, F-75006 Paris, France.

\section{Outdoor man}

\section{Eco Homo: How the Human Being Emerged from the Cataclysmic History of the Earth}

by Noel T. Boaz

BasicBooks: 1997. Pp. 278. \$25

The Aquatic Ape Hypothesis

Elaine Morgan

Souvenir Press: 1997. Pp. 205. £16.99

\section{lan Tattersall}

One of the trendiest recent exercises in palaeoanthropology has been the publication of books that in one way or another blame the emergence and subsequent evolution of our own dubious hominid lineage on the climatic oscillations of the Pleistocene epoch (the ice ages') and earlier.

So far, so good; for although some would argue long and hard about claimed correlations of climatic and ecological events with particular innovations in the hominid record, I think I'm in pretty good company in finding it inconceivable that the dramatic worldwide warmings and coolings of the Pleistocene, and the sea-level (hence geographic) changes that accompanied them, did not radically affect the course of hominid evolution. What I find truly remarkable, though, is how little these books have in common beyond the shared conviction that human evolution over the past two or three million years has been driven by the vicissitudes of climate.

Noel T. Boaz's latest offering is a case in point. Most authors on this subject find a congenial fit between a geological record of unstable climates and geographies and the notion of evolution by punctuated equilibria ('fits and starts'). Boaz, however, explicitly dismisses this evolutionary model on the rather odd and unexplained grounds of 'vitalism' and a 'lack of clear causative association in nature'. He prefers an older model of continuous evolution with varying rates, the background rate dictated by the 'Red Queen' model ('running' to stay in the same place owing to competition) and faster change coming at times of dramatic climatic shift. Unfortunately, this choice doesn't help his argument: the Pleistocene record is one of almost ceaseless environmental oscillation, so his strictly adaptationist perspective forces him to seek many more evolutionary correlates of climatic change than other authors have been comfortable with. What is more, the choice may also account for his curious neglect of the major lineageindependent 'turnover pulses' that are a central feature of the faunal history of the Plio-Pleistocene.

Boaz covers a lot of ground, beginning his story in the Miocene, well before the origin of hominids, and continuing through the first australopiths and early Homo to the first Europeans and Asians and eventually to the rise and demise of the Neanderthals and their replacement by modern humans. Although selective, this is a competent enough survey. But it shows how hard it is for Boaz to reconcile his preferred evolutionary model with his desire to tie in virtually all human evolutionary developments with ecological cataclysms. In turn, his emphasis on the primacy of ecological change collides with his reluctance to recognize more than the smallest possible number of extinct hominid species at a time when conditions for speciation could not have been more propitious. These tensions are never resolved.

Elaine Morgan is another strict adaptationist who should, in theory, get along well with Boaz. At least until she reads in his book that humans are not well adapted for swimming. Morgan, as is well known, is the foremost if not the only active proponent of the 'aquatic ape theory', which posits human descent from at least a semi-aquatic ancestry. Over the decades since The Descent of Woman was published, Morgan has honed her writing and diplomatic skills, and this fourth book in her series is an engaging presentation of a scaled-down hypothesis that simply asks that we should seriously consider that a few million years of at least semi-water-adapted ancestry might be responsible for some of our anatomical features.

Only Morgan acolytes will warm to this appeal; but along the way she takes liberal swipes (some better aimed than others) at a whole range of more orthodox views, and clearly has a good time doing so. As she has noticed, palaeoanthropologists are fond of telling each other 'Just-So' stories; and once in a while a little needling of this kind does no harm at all.

Ian Tattersall is in the Department of Anthropology, American Museum of Natural History, New York, New York 10024, USA.

The second edition of Bones of Contention: Controversies in the Search for Human Origins by Roger Lewin is now available in paperback. In a new afterword, the author looks at the way in which palaeoanthropology, while becoming more scientific, remains contentious. "Lewin may have contributed more to the evolution of a science than he had dared hope," wrote C. K. Brain in a review in Nature $(330,277 ; 1987)$. University of Chicago Press, $\$ 18.95, \mathfrak{E} 15.25$. 